\title{
Mechanical Fault Diagnosis of an On-Load Tap Changer by Applying Cuckoo Search Algorithm-Based Fuzzy Weighted Least Squares Support Vector Machine
}

\author{
Shuguo Gao $\mathbb{D}^{1},{ }^{1}$ Cong Zhou $\mathbb{D}^{2},{ }^{2}$ Zhigang Zhang, ${ }^{3}$ Jianghai Geng, ${ }^{4}$ Ruidong He, ${ }^{3}$ \\ Qingdong Yin, ${ }^{1}$ and Chao Xing ${ }^{1}$ \\ ${ }^{1}$ State Grid Hebei Electric Power Research Institute, Shjiazhuang 050021, China \\ ${ }^{2}$ State Grid Tianjin Electric Power Company, Tianjin 300010, China \\ ${ }^{3}$ State Grid Hebei Electric Power Supply Company Limited, Shjiazhuang 050000, China \\ ${ }^{4}$ Hebei Provincial Key Laboratory of Power Transmission Equipment Security Defense, North China Electric Power University, \\ Baoding 071003, China
}

Correspondence should be addressed to Cong Zhou; hd15176236798@163.com

Received 13 February 2020; Revised 12 April 2020; Accepted 18 April 2020; Published 7 May 2020

Academic Editor: Francisco J. Montáns

Copyright (C) 2020 Shuguo Gao et al. This is an open access article distributed under the Creative Commons Attribution License, which permits unrestricted use, distribution, and reproduction in any medium, provided the original work is properly cited.

To improve adaptability, feature resolution, and identification accuracy when diagnosing mechanical faults in an on-load tap changer (OLTC) of a transformer, in the present research, wavelet packet energy entropy is used to describe the information comprising vibration signal in the switch process of an OLTC, and a fuzzy weighted least squares support vector machine (CSAfuzzy weighted LSSVM) model based on the cuckoo search algorithm is proposed to identify mechanical fault types. Specifically, according to the different importance of the sample data in different periods, the idea of fuzzy weighting of training samples is proposed. The cuckoo search algorithm is used to optimise regularisation parameters, kernel function width, and weight control factor of CSA-fuzzy weighted LSSVM. Finally, the real experimental platform for typical mechanical faults of an OLTC is established, and the vibration signals of several typical mechanical faults under different degrees of fatigue are obtained. The results show that the new method achieves a higher accuracy rate of fault identification compared with other common methods. It can better deal with small sample and nonlinear prediction problems and shows higher fitting accuracy than CSA-LSSVM, single LSSVM, and radial basis neural network methods and is thus better suited for mechanical fault diagnosis in OLTCs. This paper presents a new intelligent diagnosis scheme for mechanical faults of on-load tap changers, which can achieve noninterruption and nonintrusive detection. The proposed diagnosis method would change the traditional diagnosis method of the on-load tap changer and improves the power supply quality and the detection efficiency under the premise of ensuring the safety of the staff.

\section{Introduction}

As important electrical equipment in the power transmission and distribution network in China, an on-load tap changing transformer (OLTCT) mainly plays an important role in connecting high- and low-voltage power transmission and distribution network, regulating and controlling power flows, and stabilising the voltage at the load centre of a system. OLTCTs have been increasingly extensively applied in modern power transmission and distribution networks in China. The voltage regulation function of an OLTCT is realised by switching the tap position of contacts of an onload tap changer (OLTC) step-by-step. The operating condition of an OLTC affects the safe and reliable operation of a power system and also plays an important role in guaranteeing flexible dispatching of power grids; however, the manufacturing and maintenance technologies for OLTCs have not yet been perfected. The accidents caused by faults in OLTCs happen frequently with the large-scale application of OLTCTs in power grids. Online monitoring and diagnosis of mechanical faults of OLTCs play an increasingly important role in modern power transmission 
and distribution network, also exert excellent practical significance on the overall safe operation of power grids, and are regarded as the important demand of construction of ubiquitous power for the Internet of Things.

Owing to the vibration signals of OLTCs presenting time-varying characteristics and instability, some time-domain analysis methods (such as fast Fourier transform (FFT)) show unsatisfactory performance when they are used to detect certain faults. By introducing wavelet transform into mechanical fault detection of OLTCs, Kang and Birtwhistle $[1,2]$ established a ridge distribution map to characterise the mechanical condition of OLTCs by utilising the wavelet coefficient obtained through two-dimensional discrete wavelet transform. The ridge distribution map clearly reveals the disparity of features of OLTCs with mechanical faults and in normal state. Kang and Birtwhistle and Rivas et al. $[3,4]$ extracted the time-domain features (including peak time and amplitude) of vibration signals by applying wavelet analysis and compared the features of vibration signals in normal and fault states by using a self-organising map and genetic algorithm (GA). On this basis, a database for features of vibration signals of OLTCs is constructed to establish a set of evaluation procedure for the mechanical state of OLTCs; however, only low-frequency components are selected for further decomposition during each calculation in wavelet analysis and therefore wavelet analysis fails to extract the features of high-frequency signals. The highfrequency sections of vibration signals of OLTCs contain lots of effective information, so it is necessary to make certain improvements to the wavelet analysis technique.

As the further extension of wavelet analysis, wavelet packet analysis can perform multilevel and multiresolution decomposition of low- and high-frequency components of vibration signals, which can deal with the time-varying, nonstationary vibration signals arising from OLTCs. Li et al. [5] in Shandong University extracted the time- and frequency-domain features of OLTC vibration signals by employing wavelet packet decomposition. After collecting the vibration signals on the OLTC surface by applying a piezoelectric accelerometer, the frequency composition of OLTC vibration signals at different scales can be attained through wavelet packet decomposition. On the basis of effectively decomposing the mechanical vibration signals of OLTCs, the dynamic characteristics contained therein can be reasonably described.

The common methods for diagnosing fault modes mainly include artificial neural network (ANN) and support vector machine in the field of fault diagnosis. Shi [6] established a backpropagation neural network optimised by adaptive genetic algorithm (AGA) to diagnose the mechanical fault types of OLTCs; however, a neural network requires a large training sample dataset and is likely to be trapped in a local optimum. By utilising a fuzzy weighted least square support vector machine (LSSVM) model based on particle swarm optimisation (PSO), Kong et al. [7] diagnosed the mechanical faults of operating mechanisms of a high-voltage circuit breaker. The model could provide good fitting accuracy based on a small training dataset and avoid the occurrence of local optima. The mechanical state of
OLTCs changes during long-term operation, and the features of vibration signals measured in different periods also gradually change; therefore, the characteristic data of vibration signals of OLTCs in different periods have varying importance in the training process of the model. Thus, a fuzzy weighted LSSVM model is introduced to assign varying weights to different characteristic parameters obtained through feature extraction of vibration signals. Additionally, the regularisation parameters, kernel function width, and weight control factor of the CSA-fuzzy weighted LSSVM are optimised by applying the cuckoo search algorithm (CSA) to improve the fitting accuracy of the model, thus increasing the accuracy of diagnosis of mechanical faults in OLTCs.

To improve the adaptability, feature resolution, and fitting accuracy of the model for diagnosing mechanical faults in OLTCs, the information contained in vibration signals in the switchover process of OLTCs is described by using wavelet packet energy entropy. Additionally, the fuzzy weighted LSSVM optimised by CSA is proposed for use when identifying mechanical fault types in OLTCs. Moreover, based on an SYJZZ-35 OLTC, a real model experimental platform for typical mechanical faults of an OLTC is built to attain the vibration signals of several typical mechanical faults (such as loose moving contact, loose transition contact, and a motor shaft jam). Moreover, the proposed method for diagnosing mechanical faults of OLTCs is used to identify the mechanical states of OLTCs corresponding to vibration signals and compared with the existing methods to verify its effectiveness. This paper presents a new intelligent diagnosis scheme for mechanical faults of on-load tap changers, which can achieve noninterruption and nonintrusive detection. The proposed diagnosis method would change the traditional diagnosis method of the on-load tap changer and improve the power supply quality and the detection efficiency under the premise of ensuring the safety of the staff.

\section{Diagnosis of Mechanical Faults in OLTCs Based on CSA-Fuzzy Weighted LSSVM}

2.1. Wavelet Packet Transform and Wavelet Packet Energy Entropy. A wavelet packet can accurately and comprehensively deal with high- and low-frequency signals, so it plays an important role in improving the time-frequency resolution of signals and exploring features of full-band signals. Therefore, wavelet packet decomposition can be used to process vibration signals arising from mechanical faults on OLTCs.

$h_{k}$ and $g_{k}$ refer to the coefficients in filters of wavelet packet transform; moreover, the wavelet function is defined as $\phi(t)$, and the corresponding scaling function is expressed as $y(t)$. The wavelet function and scaling function can be expressed by using an equation set as follows:

$$
\left\{\begin{array}{l}
\phi(t)=\sum_{k=Z} h_{k} \phi(2 t-k), \\
y(t)=\sum_{k=Z} g_{k} \phi(2 t-k) .
\end{array}\right.
$$


The following function sets are defined:

$$
\left\{\begin{array}{l}
u_{2 n}(t)=\sqrt{2} \sum_{k=Z} h(k) \phi_{n}(2 t-k), \\
u_{2 n+1}(t)=\sqrt{2} \sum_{k=Z} g(k) u_{n}(2 t-k),
\end{array}\right.
$$

where $u_{2 n}(t)$ and $u_{2 n+1}(t)$ separately represent the scaling and wavelet functions and the function set $\left\{u_{n}\right\}$ is a wavelet packet defined by the basis function $u_{0}(t)$. Thus, the wavelet packet can be regarded as a set of a class of functions composed of wavelet and scaling functions.

Hence, the function set $\left\{2^{j / 2} u_{n}\left(2^{j} t-k\right) ; n \in k ; k \in Z\right\}$ can be attained, where $j, k$, and $n$ refer to the scaling function, displacement function, and oscillating coefficient, respectively. By adjusting the values of $j$ and $k$, wavelet packet decomposition can be applied to mechanical vibration signals from OLTCs.

The basic principle underlying frequency division of wavelet packet decomposition is described in Figure 1. In short, wavelet packet decomposition can be used to analyse the vibration signals by constantly conducting high- and low-frequency decomposition of vibration signals [7].

In Figure 1, $L$ represents the low-frequency part, showing low-pass characteristics; $H$ denotes the high-frequency part, with high-pass characteristics; the first digit of the subscript represents the level of wavelet packet decomposition.

It is supposed that $u$ stands for an original signal. After undergoing $J$ layers of wavelet packet decomposition, $2 J$ subsignals that are not overlapped can be attained and serve as the characteristic signals of the originals. It is supposed that the reconstructed signal at the $i^{\text {th }}$ node in the layer $J$ is $u_{i}$, and thus, the corresponding Shannon entropy is calculated as follows:

$$
E\left(u_{i}\right)=-\sum_{i} u_{i}^{2} \log \left(u_{i}^{2}\right)
$$

An eigenvector is constructed by using the energy of $2 J$ nodes, which can be used as the input of the subsequent classifier.

The data of each eigenvector are normalised to $[0,1]$ using the following formula: $x_{i}^{*}=\left(x_{i}-x_{\min }\right)$ / $\left(x_{\max }-x_{\min }\right)$. Here, $x_{i}$ refers to the $i^{\text {th }}$ datum of an eigenvector; $x_{\min }$ and $x_{\max }$ denote the minimum and maximum of the eigenvector; and $x_{i}^{*}$ represents the normalised value, respectively. In this way, the data pertaining to various eigenvectors can be mapped into $[0,1]$.

2.2. Fuzzy Weighted LSSVM. In the fields of pattern recognition, knowledge engineering, management decisionmaking, and social selection, it is a very important issue that people estimate the various factors of complex things to make decisions. For example, in terms of OTLC mechanical condition assessment, the influence of sampling data in different periods on the assessment results is different, so different periods of data must be given different weights to highlight the factors that have a greater impact on the assessment. Because the process of OLTC mechanical condition evaluation itself has a certain degree of ambiguity,

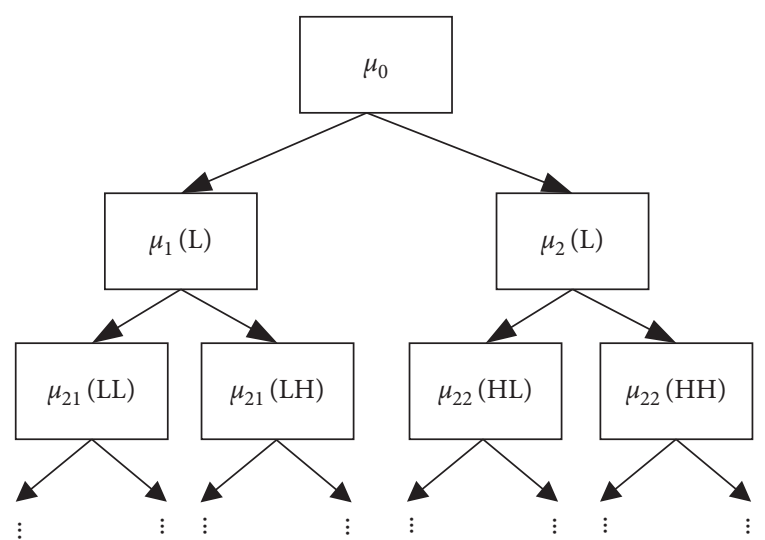

FIgURE 1: Frequency division of wavelet packet decomposition.

there is one kind of ambiguity in subjective cognitive ability. Therefore, the diagnosis process of OLTC mechanical failure becomes a thinking process with a lot of ambiguity, and it is necessary to assign a weight to different factors to obtain a better diagnosis effect. In this case, it is a good method to use fuzzy mathematics theory to fuzzy-weight the training data in different periods. The effect of this method has been confirmed in many previous studies [8-10].

Given a sample set $T_{g}=\left(x_{i}, y_{i}\right)$ (where $M$-dimensional input $x_{i} \in \mathbf{R}^{\mathrm{M}}$ and one-dimensional output $y_{i} \in \mathbf{R}, i=$ $1,2, \cdots, N)$, it is feasible to map the nonlinear, indecomposable samples into high-dimensional characteristic space by utilising nonlinear mapping function $\varphi(x)$, so as to transform the estimation problem of nonlinear function into a linear regression problem:

$$
y(x)=\omega^{T} \varphi(x)+b .
$$

According to the structural risk minimisation principle [11], the linear regression problem can be rendered equivalent to an optimisation problem with equality constraint:

$$
\begin{aligned}
& \min _{\omega, b, e} \quad J(\omega, e)=\frac{1}{2} \omega^{T} \omega+\frac{C}{2} \sum_{i-1}^{N} e_{i}^{2}, \\
& \text { s.t. } \quad y_{i}=\omega^{T} \varphi\left(x_{i}\right)+b+e_{i}, \quad i=1,2, \cdots, N .
\end{aligned}
$$

Afterwards, the above constrained optimisation problem can be transformed into an unconstrained problem by using Lagrange multipliers:

$$
L(\omega, b, e, a)=J(\omega, e)-\sum_{i-1}^{N} \alpha_{i}\left(\omega^{T} \varphi\left(x_{i}\right)+b+e_{i}-y_{i}\right)
$$

where $\alpha_{i}(i=1,2, \cdots, N)$ refers to a Lagrange multiplier.

According to the KKT condition [11], the partial derivatives of various variables are calculated based on $L(\omega, b, e, a)$ for the unconstrained optimisation problem. Let the derivative function be 0 , and the extreme point of $L(\omega, b, e, a)$ can be determined. Therefore, the above unconstrained optimisation problem can be transformed into

$$
\left[\begin{array}{cc}
0 & \mathbf{I}^{T} \\
\mathbf{I} & \mathbf{\Omega}+\mathbf{C}^{-1} \mathbf{I}
\end{array}\right]\left[\begin{array}{l}
\mathbf{b} \\
\mathbf{a}
\end{array}\right]=\left[\begin{array}{l}
0 \\
\mathbf{y}
\end{array}\right]
$$


where $\quad \mathbf{I}=[1,1, \cdots, 1]^{T} ; \quad \mathbf{y}=\left[y_{1}, y_{2}, \ldots, y_{N}\right]^{T} ; \quad \alpha=$ $\left[\alpha_{1}, \alpha_{2}, \cdots, \alpha_{N}\right]^{T} ; \Omega=\left\{\Omega_{k l} \mid k, l=1,2, \cdots, N\right\}$, where $\Omega_{k l}=$ $\varphi\left(x_{k}\right)^{T} \varphi\left(x_{l}\right)=K\left(x_{k}, x_{l}\right), \quad k, l=1,2, \cdots, N ;$ and $K\left(x_{k}, x_{l}\right)$ refers to the kernel function of support vector machine. The radial basis function (RBF) is applied for the calculation: $K\left(x_{k}, x_{l}\right)=\exp \left(-x_{k}-x_{l}^{2} / \sigma^{2}\right)$.

Linear equation set (8) is calculated to attain $\mathbf{a}$ and $\mathbf{b}$, that is, the output of the LASVM can be obtained:

$$
y(x)=\sum_{i=1}^{N} \alpha_{i} K\left(x, x_{i}\right)+b .
$$

The training effect and generalisation ability of the LSSVM model are affected by the parameter $\sigma$ in the kernel function and penalty factor $C$. Hence, CSA is used to optimise $\sigma$ and $C$ to eliminate the effect of subjective factors on the reliability of the proposed model.

The mechanical state of an OLTC gradually changes during long-term operation, and the features of vibration signals measured over different periods also vary gradually. The measured latest data of vibration signals can better reflect the current mechanical state of the OLTC; therefore, the importance of feature data of vibration signals of OLTC changes in different periods in the training process of the model; the latest data are more important; therefore, it is feasible to add a fuzzy weighted membership degree $\mu_{i}$ to each sample vector in the training sample set. Thus, the optimisation problem in equation (5) can be corrected to

$$
\min _{\omega, b, e} J(\omega, e)=\frac{1}{2} \omega^{T} \omega+\frac{C}{2} \sum_{i=1}^{N} \mu_{i} e_{i}^{2} .
$$

If the sample size of the training sample set is $N$, the corresponding fuzzy weighted membership degree $\mu_{i}$ of the $i^{\text {th }}$ group of samples can be defined using equation (11) according to the principle that the latest sample is of greater importance:

$$
\mu_{i}=f(i)=(1-\varepsilon) \sqrt{\frac{i-1}{N-1}}+\varepsilon,
$$

where $\varepsilon \in[0,1]$ denotes the weight control factor, $\varepsilon=\mu_{i} \leq \cdots \leq \mu_{N}=1$; that is, the lower the value of $\varepsilon$, the less important the early data in the training of the model.

2.3. CSA. As a new bionic swarm evolutionary algorithm, CSA was proposed by Yang and Deb $[12,13]$ in the University of Cambridge by using the Levy flight search principle on the basis of exploring the reproductive mechanism of nest parasitism among cuckoos. Through plenty of tests, the algorithm shows better performance in many aspects compared with GA and PSO [14]. For example, CSA yields better global search effect while setting a smaller number of parameters and has a rapid convergence rate, with superior generalisation ability and stronger robustness. Therefore, CSA has been widely used in many studies [15-18].

The optimisation mode of CSA learns from the breeding habits of cuckoos. Cuckoos will lay eggs in nests of other birds while they do not incubate the eggs. The owner of such a nest is called the host bird. If the host bird finds that an egg in the nest is not its own, the egg, or nest, will be abandoned. Thus, the cuckoo egg fails to be successfully incubated. Hence, cuckoos prefer to choose a nest where a host bird just lays eggs. Once the cuckoo egg can be preserved, the fledgling cuckoo can push the other eggs out of the nest instinctively owing to a cuckoo egg exhibiting a higher incubation rate. In such a way, the host bird will raise the fledgling cuckoo egg as its own offspring. Additionally, the flight path of cuckoos when searching for their own nests can be described by using Levy flight concepts [19]. Therefore, CSA usually performs optimisation through the following two paths: (1) local search based on the probability that the cuckoo eggs are identified by the host bird; (2) global search by determining the step size according to Levy flight concepts.

The local search mode of CSA can be expressed as follows:

$$
x_{i}^{(t+1)}=x_{i}^{t}+\alpha s \otimes H\left(p_{0}-\delta\right) \otimes\left(x_{j}^{t}-x_{k}^{t}\right),
$$

where $x_{j}^{t}$ and $x_{k}^{t}$ denote two different series; $H(u), \delta, s, p_{a}$, and $\alpha$ refer to the Heaviside function, a random number, the step size, the probability that cuckoo eggs are found by a host bird, and the parameter for controlling the step size, respectively.

The global search mode of CSA can be expressed as

$$
x_{i}^{(t+1)}=x_{i}^{t}+\alpha \otimes L(s, \lambda),
$$

where $L(s, \lambda)=\lambda \Gamma(\lambda) \sin (\pi \lambda / 2) / \pi 1 / s^{1+\lambda}, s \gg s_{0} \quad(1<\lambda \leq 3)$, and $\alpha$ represents the parameter for controlling the step size. Similar to PSO, CSA also employs scalar product " $\otimes$ "; however, CSA which performs a random search based on Levy flight and thus requires a larger step size; therefore, CSA returns a faster convergence rate and can realise better optimisation in tandem with a local search.

The specific steps in diagnosis of mechanical faults of an OLTC by applying the CSA-fuzzy weighted LSSVM are as follows:

(1) The historical data of vibration signals of the OLTC are collected or a fatigue test is conducted on the OLTC to simulate the change in its working state during long-term operation and attain the vibration signals in the switchover process in different operating periods. Moreover, the wavelet packet energy entropy is obtained through wavelet packet decomposition, serving as the input variable of the fuzzy weighted LSSVM, and its corresponding mechanical state type of the OLTC is taken as the output variable. Furthermore, a training sample set is formed by using data from different periods.

(2) Initialisation of populations: $n$ bird nests $X_{0}=\left(x_{1}^{(0)}, x_{2}^{(0)}, \ldots, x_{n}^{(0)}\right)^{T}$, where $x_{i}^{(0)}=\left(\sigma_{i}^{(0)}, C_{i}^{(0)}\right.$, $\left.\ldots, \varepsilon_{i}^{(0)}\right)^{T}(i=1,2, \cdots, n)$, are stochastically generated. The root mean square error (RMSE) is applied as the fitness function, which is expressed as follows:

$$
\mathrm{RMSE}=\sqrt{\frac{1}{N} \sum_{i=1}^{N}\left(y_{t}-y_{t}^{*}\right)^{2}}
$$


where $y_{t}$ refers to the predicted value obtained by using the fuzzy weighted LSSVM at time $t$, which is the corresponding actual value in the training sample set at time $t$. The fitness of each bird nest is calculated to find the current optimal position $x_{b}^{(0)}$ of the nest.

After obtaining the sample data, the overall flow of the diagnostic method is shown in Figure 2.

(3) Location update: the optimal position $x_{b}^{(0)}$ of the nest in the last generation is retained and the positions $X_{j}=\left(x_{1}^{(j)}, x_{2}^{(j)}, \cdots, x_{n}^{(j)}\right)^{T}$, where $x_{i}^{(j)}=\left(\sigma_{i}^{(j)}, C_{i}^{(j)}\right.$, $\left.\varepsilon_{i}^{(j)}\right)^{T}(i=1,2, \cdots, n)$, of $n$ bird nests are updated according to Levy flight mode. Furthermore, the fitness of the positions of each bird nest is calculated to find the optimal position $x_{b}^{(t)}$, which is compared with the fitness of the optimal position $x_{b}^{(0)}$ of the bird nests in the last generation. If the former is superior, the position is updated again according to the Levy flight mode; otherwise, the optimal position $x_{b}^{(0)}$ of the nests in the last generation is retained.

(4) Local optimisation: random number $\delta \in(0,1)$ is generated and compared with $P_{a}$. If $r>P_{a}$, the original position of the nests is retained; if $\delta<P_{a}$, the position of the nests is updated using a stochastic step size; afterwards, the fitness of the position of the nests is separately calculated and compared with that of their original positions. If the new position of the nests has a higher fitness, the position is updated again in the same way; otherwise, the position of the nests in the last generation is retained to attain the positions $X_{i+1}=\left(x_{1}^{(j+1)}, x_{2}^{(j+1)}, \cdots, x_{n}^{(j+1)}\right)$ of $n$ nests after updating.

(5) Steps (3) and (4) are repeated until reaching the preset maximum iteration number. The optimal position $x_{b}=\left(\sigma_{b}, C_{b}, \varepsilon_{b}\right)^{T}$ of the nests obtained through multiple iterations is taken as the preset parameter of the fuzzy weighted LSSVM.

\section{Real Model Experiment on Mechanical Faults in an OLTC}

3.1. Real Model of an Experimental OLTC. The typical defect model for an OLTC of a transformer is established by selecting the SYJZZ-35 OLTC (Figure 3), based on which several common mechanical faults (such as a worn contact, loose fixed contact, and a contact jam) are simulated. The three-phase SYJZZ-35 OLTC adopted jumper voltage regulation in the middle part, with direct switching, a rated voltage of $35 \mathrm{kV}$, and seven working positions. This type of OLTC, with its simple structure, operates reliably and is easy to disassemble and maintain, so it is applicable for the present research on mechanical fault diagnosis on OLTCs.

3.2. Real Model Experiment for the Typical Mechanical Faults in an OLTC. To test the effect of the proposed method for mechanical faults of OLTCs on fault identification, the simulation experiment of faults is conducted using the typical defect model for an OLTC by taking looseness and jamming faults as examples. According to the statistical reporting of field data, it can be seen that looseness and jamming faults accounted for a proportion greater than $60 \%$ among the mechanical faults of the OLTC. Therefore, the acquired sample data are deemed representative. When simulating the faults, the loose contacts are simulated by unscrewing the fastening screw or spring of the contacts. A jammed motor is simulated by applying resistance to the motor shaft, as shown in Figures 4 and 5. To simulate the change of fatigue state of OLTCs during long-term operation, the looseness of the fastening screw and spring is increased step-by-step when simulating loose contacts; the resistance on the motor shaft is also increased step-by-step when simulating the jamming of the driving mechanism. The time stamps of different experimental groups are separately defined to simulate the sample data measured in different periods. By doing so, the superiorities of the fuzzy weighted LSSVM relative to the LSSVM can be validated.

Three UTL2001X piezoelectric acceleration sensors (sensitivity: $500 \mathrm{mV} / \mathrm{g}$ ) produced by Beijing Quatech Electronic Co., Ltd (Beijing, China) are used. The vibration signals in the switchover process of the OLTC are acquired by employing a DI-4108 data acquisition system produced by DATAQ Co., Ltd (USA). During the experiment, three vibration sensors are distributed as shown in Figure 6 to record the mechanical vibration signals in the switchover process of OLTCs in three interactively vertical directions.

To reduce the random error in the switchover process of the OLTC, it is switched over all tap positions in the experiment; that is, the OLTC is switched over from the first to the seventh tap position (uplink) and then reversely switched over to the first tap position (downlink). Additionally, for loose contacts, these are separately simulated at each of the three phases of the OLTC by considering the effect of faults at different positions on the collected vibration signals.

\section{Analysis and Results}

4.1. Acquisition of Wavelet Packet Energy Entropy of Vibration Signals. The wavelet packet transform is characterised by multiresolution analysis and imposes no requirement on stability [20]. Therefore, the composition information of vibration signals at different frequencies can be attained in various scale spaces after the collected mechanical vibration signals of the OLTC are decomposed by using a wavelet packet transform. While analysing the mechanical vibration signals, the dynamic characteristics thereof are accurately described.

Based on wavelet packet decomposition, it is necessary to calculate multiresolution characteristic parameters in effective frequency bands to characterise the time- and frequency-domain complexity in the mechanical vibration signals of the OLTC (Figure 7).

The multiresolution characteristic parameters (effective entropy) in effective frequency bands are extracted according to the following steps:

(1) At first, the wavelet packet decomposition is performed on the collected mechanical vibration signals 


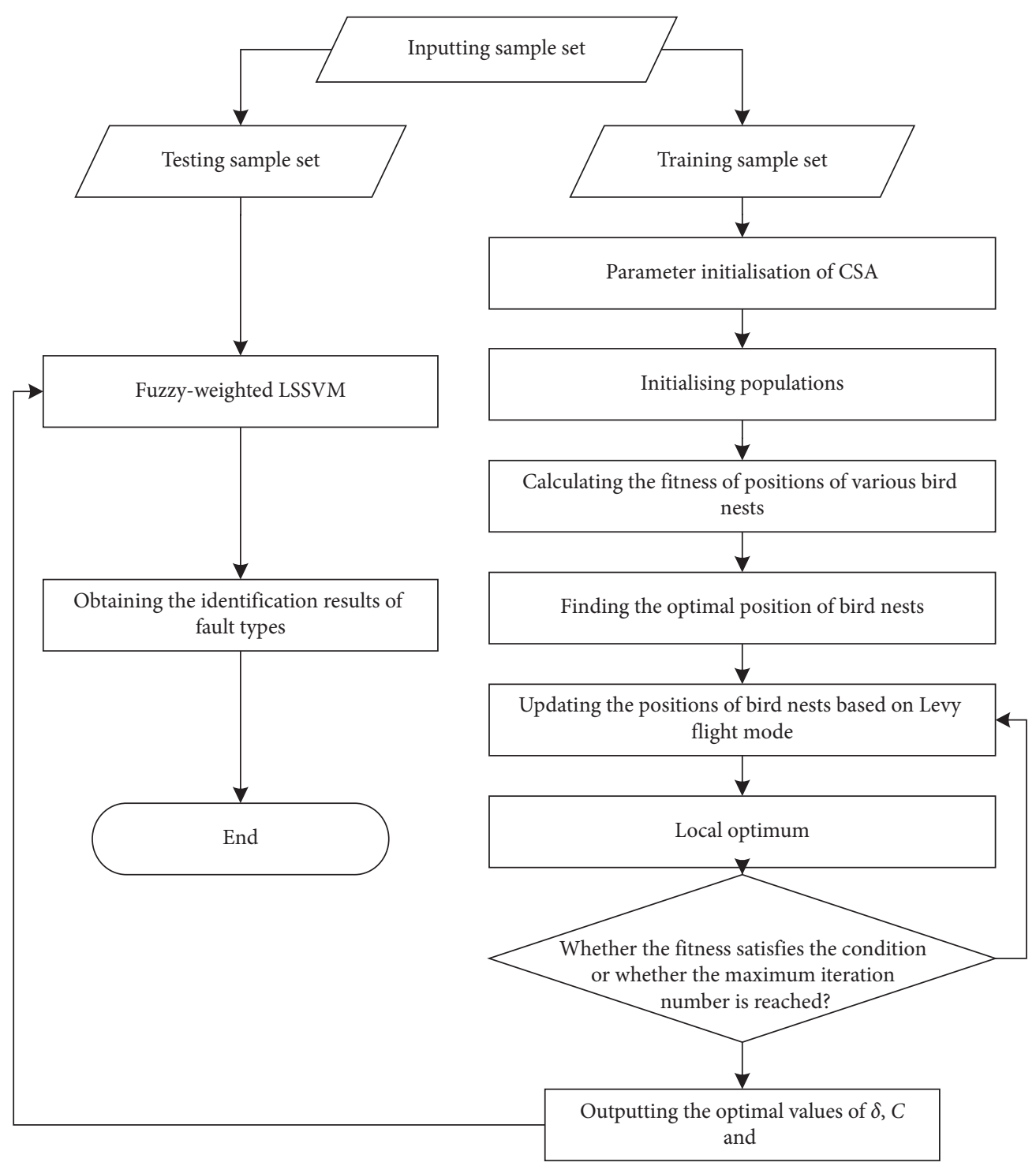

FIGURE 2: Mechanical fault diagnosis for an OLTC based on the CSA-fuzzy weighted LSSVM model.

of the OLTC to calculate the wavelet packet transforms of the mechanical vibration signals at various scales $j(j=1,2, \cdots, J)$. Furthermore, the wavelet packet coefficients at various scales are expressed as follows:

$$
\left\{\begin{array}{l}
d_{l}^{j, 2 n}=\sum_{k} h_{k-2 l} d_{k}^{j-l, n}, \\
d_{l}^{j, 2 n+1}=\sum_{k} g_{k-2 l} d_{k}^{j-l, n} .
\end{array}\right.
$$

When decomposing the mechanical vibration signals of OLTCs, $j=3$; the mechanical vibration signals of OLTCs are decomposed into eight frequency bands, which are separately expressed as $\left[S_{3,0}\right],\left[S_{3,1}\right], \cdots,\left[S_{3,8}\right]$, in which lowfrequency parts (below $12.5 \mathrm{kHz}$ ) correspond to four frequency bands $[3,0],[3,1],[3,2]$, and $[3,3]$ while the highfrequency parts correspond to the remainder.
(2) Threshold processing: the wavelet packet coefficients corresponding to low-frequency parts are processed according to the threshold to retain only those coefficients exceeding the threshold, so as to eliminate the low-frequency noise components contained in vibration signals. To be specific,

$$
x(\widehat{i})= \begin{cases}x(i), & |x(i)|>l, \\ 0, & |x(i)| \leq l\end{cases}
$$

where $l$ represents the threshold, which can be calculated according to $l=n \delta$, where $n$ refers to a constant, and $\delta$ denotes the standard deviation of the wavelet packet coefficient and is expressed as follows:

$$
\delta^{2}=\frac{1}{N / 2} \sum_{i=1}^{N / 2}[x(i)-\bar{x}]^{2}
$$




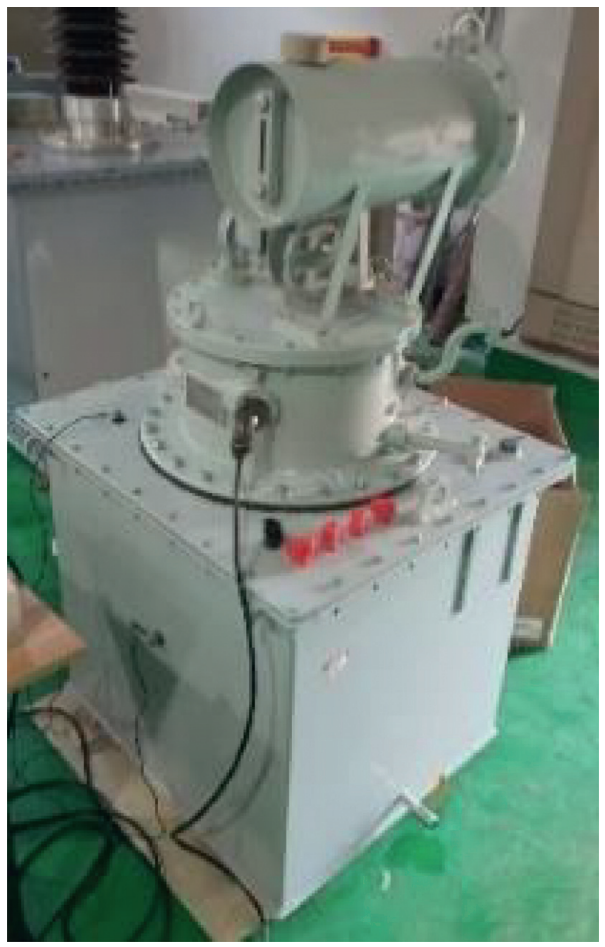

Figure 3: SYJZZ-35 OLTC.

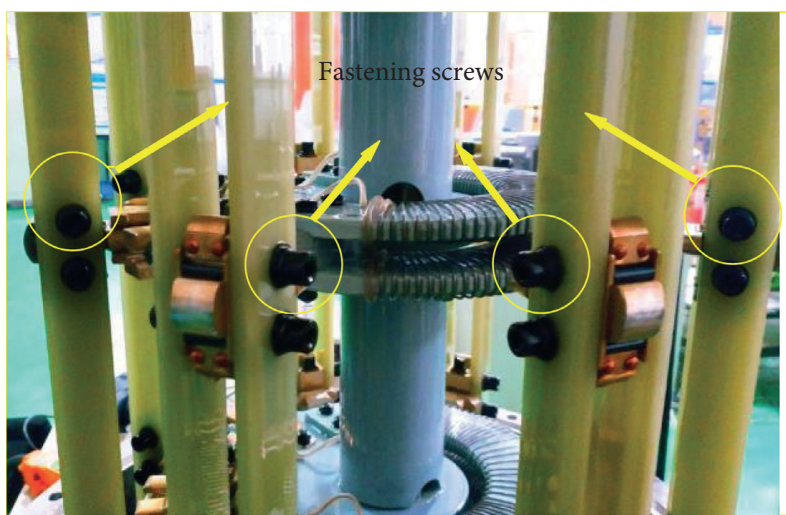

(a)

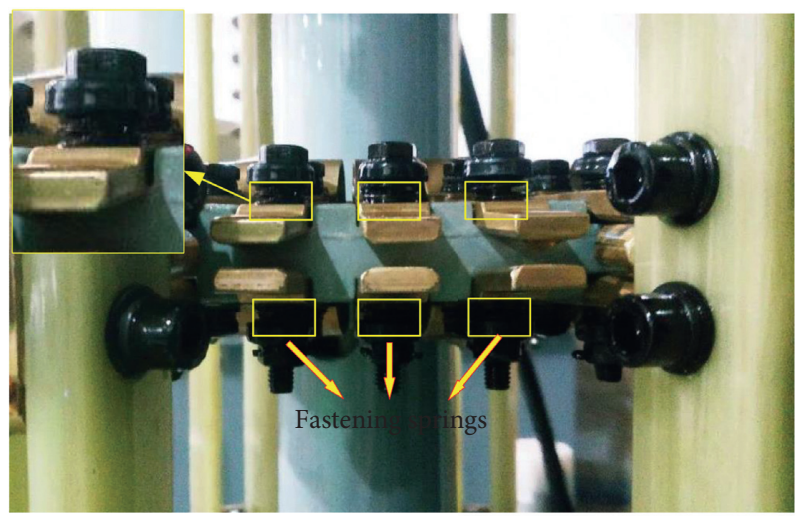

(c)

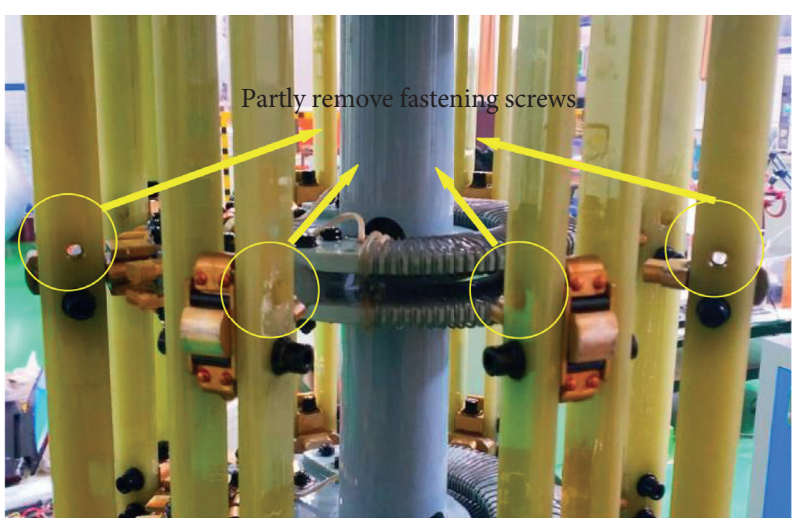

(b)

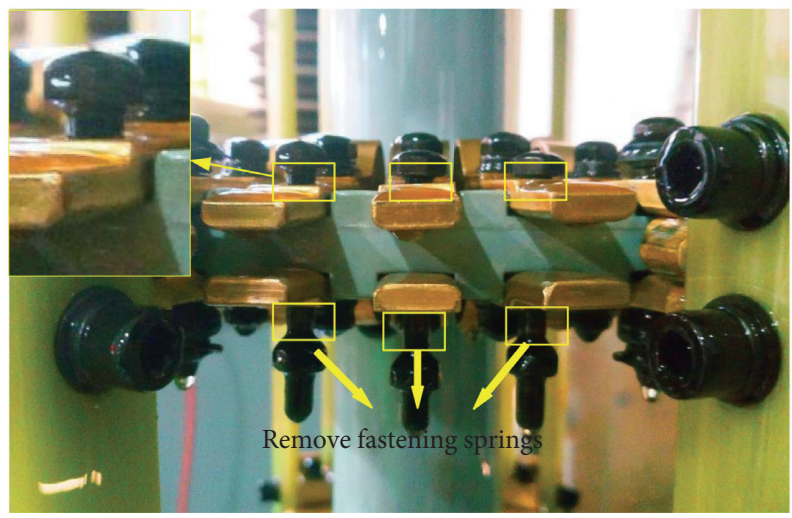

(d)

FIgURE 4: Fault simulation of typical looseness. (a) Normal working condition of moving contact. (b) Simulating the loosening of moving contact. (c) Normal working condition of transition contact. (d) Simulating the loosening of transition contact. 


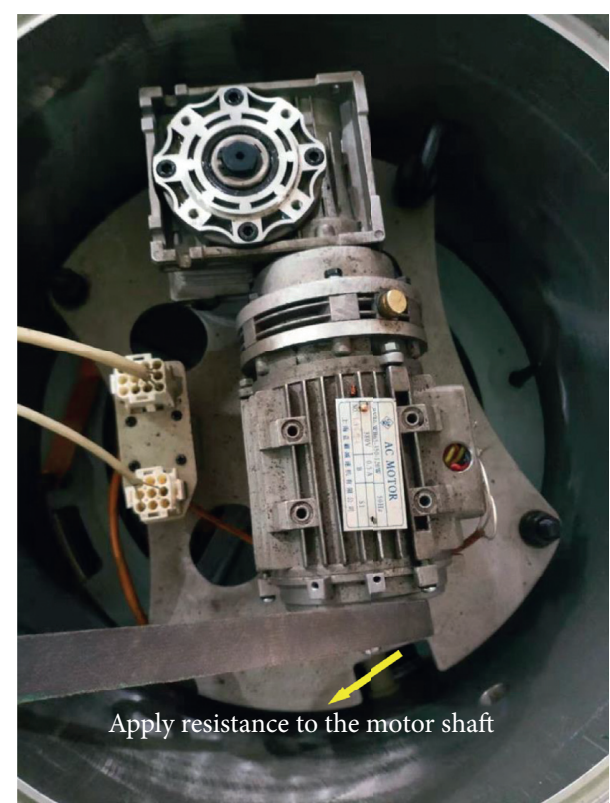

FIgURE 5: Fault simulation of motor jamming.

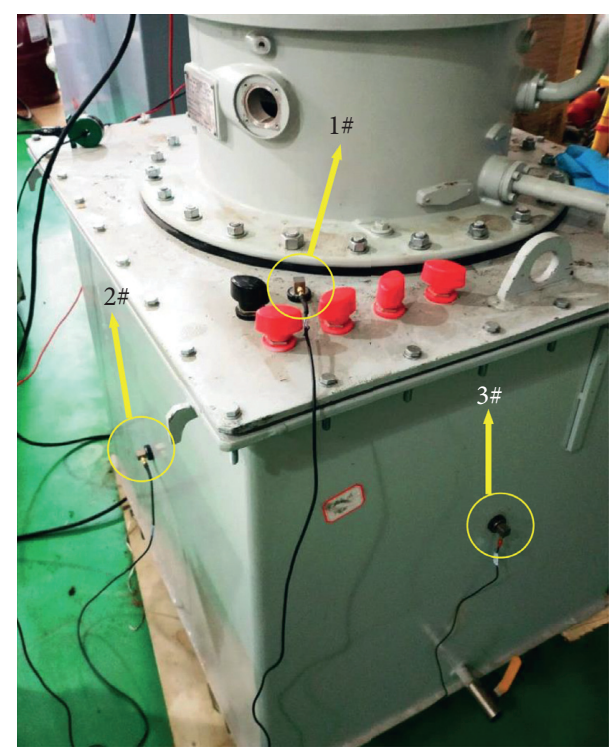

Figure 6: Positions of accelerometers.

where $\bar{x}$ and $N$ denote the mean and the sequence length of mechanical vibration signals, respectively.

(3) Acquisition of energy entropy: the signals collected from various nodes $\left[S_{3,0}\right],\left[S_{3,1}\right], \cdots,\left[S_{3,8}\right]$ are reconstructed to calculate Shannon entropies of various nodes. Eventually, the effective entropy for characterising the mechanical vibration signals of the OLTC is obtained through combination thereof.

4.2. Verification of the Effect of the Model in Fault Identification. In the present study, 330 groups of data under four working conditions (normal working conditions, loose moving contact, loose transition contact, and a jammed driving mechanism) are selected as training samples.

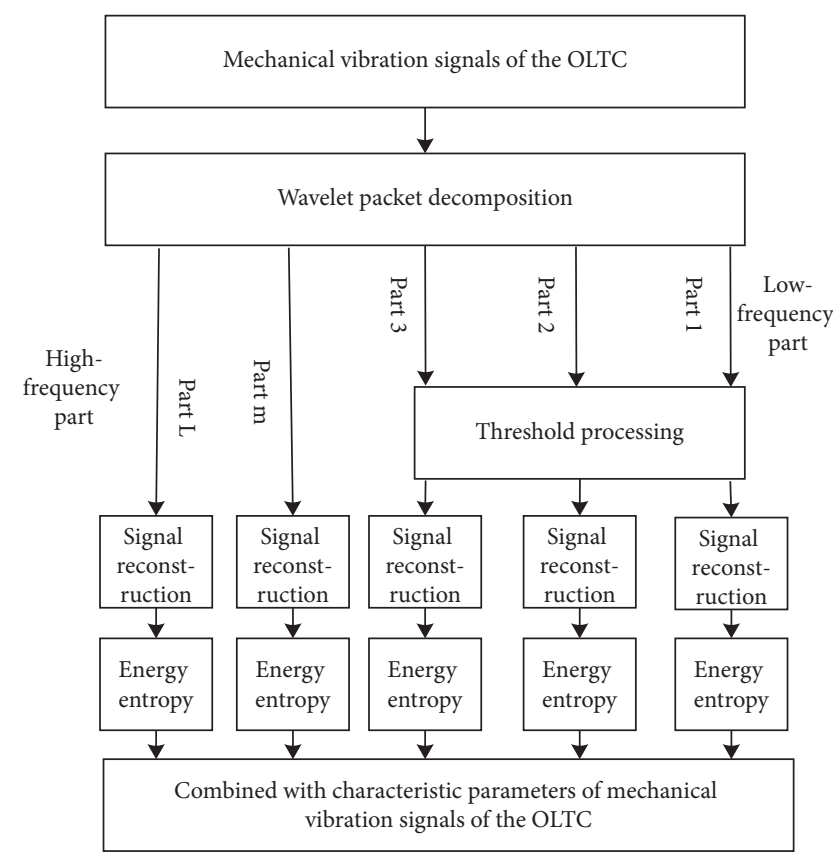

FIgURE 7: Extraction of characteristic parameters of vibration signals of the OLTC.

Additionally, 50 groups of data under each working condition are chosen as test samples. To verify the effect of the CSA-fuzzy weighted LSSVM model in identifying the mechanical fault types of the OLTC, single LSSVM, CSALSSVM, and RBF neural network models are introduced for comparison. Moreover, the initial parameters of single LSSVM and RBF neural network models are set as listed in Table 1.

In the CSA-fuzzy weighted LSSVM, the optimal values of regularisation parameter $C$, kernel function width $\sigma^{2}$, and weight control factor $\varepsilon$ are 979.3348, 175.5435, and 0.631, respectively. The confusion matrices obtained by processing 200 groups of test data based on the four models are shown in Tables 2-5.

The statistical analysis results of the effect of the four models in fault identification are listed in Table 6. The "kappa" coefficient is used to measure the accuracy of the models for fault identification, which represents the fitting degree between the predicted results and actual working conditions. The coefficient can better reflect the prediction and classification effect of the model relative to the percentage of accurate classifications [21] and is calculated as follows:

$$
k a p p a=\frac{N \sum_{i=0}^{c-1} a_{i i}-\sum_{i=0}^{c-1}\left(a_{i+} a_{+i}\right)}{N^{2}-\sum_{i=0}^{c-1}\left(a_{i+} a_{+i}\right)},
$$

where $a_{i i}$ refers to the value of the positive diagonal in the confusion matrix; $a_{i+}$ and $a_{+i}$ denote the sums of values in the $i^{\text {th }}$ row and $j^{\text {th }}$ column in the confusion matrix; and $N$ is the total number of the test samples.

As shown in Table 6, CSA-fuzzy weighted LSSVM presents the highest classification accuracy and the largest Kappa value, thus showing optimal accuracy of fault identification. The three prediction models based on LSSVM 
TABLE 1: Initial parameters of the models for comparison.

\begin{tabular}{lcc}
\hline Model & Parameter & Value \\
\hline \multirow{2}{*}{ Single LSSVM } & Regularisation parameter $C$ & 200 \\
& Kernel function width $\sigma^{2}$ & 90 \\
\hline & Node number in the hidden layer & 50 \\
RBF neural network & Maximum iteration number & 100 \\
& Learning rate & 0.1 \\
& Error & 0.0004 \\
\hline
\end{tabular}

TABLE 2: Confusion matrix of RBF neural network.

\begin{tabular}{cccccc}
\hline & \multicolumn{5}{c}{ Predicted working } \\
& 1 & 2 & 3 & 4 \\
\hline & 1 & 50 & 0 & 0 & 0 \\
Actual working condition & 2 & 3 & 31 & 8 & 8 \\
& 3 & 2 & 9 & 29 & 10 \\
& 4 & 2 & 10 & 11 & 27 \\
\hline
\end{tabular}

TABLE 3: Confusion matrix of single LSSVM.

\begin{tabular}{cccccc}
\hline & \multicolumn{5}{c}{$\begin{array}{c}\text { Predicted working } \\
\text { condition }\end{array}$} \\
& 1 & 2 & 3 & 4 \\
\hline \multirow{3}{*}{ Actual working condition } & 1 & 47 & 2 & 0 & 1 \\
& 2 & 2 & 43 & 3 & 2 \\
& 3 & 2 & 3 & 42 & 23 \\
& 4 & 2 & 3 & 0 & 45 \\
\hline
\end{tabular}

are successively displayed as single LSSVM, CSA-LSSVM, and CSA-fuzzy weighted LSSVM, respectively, in terms of their kappa values. This validated the suggestion that the cuckoo algorithm can optimise the LSSVM to improve its fitting accuracy. Relative to CSA-LSSVM, the classification accuracy of CSA-fuzzy weighted LSSVM is further strengthened. The $\mathrm{RBF}$ neural network is inferior to the aforementioned predictive models based on LSSVM in terms of classification accuracy. The reason for this is that an RBF neural network model exhibits poor capacity for processing small sample datasets, and therefore, it fails to attain an ideal effect unless there are a large number of training samples; however, LSSVM presents a strong ability to process small sample data, so it is better suited to identify mechanical faults in an OLTC compared with an RBF neural network.

Above all, the proposed CSA-fuzzy weighted LSSVM can deal with small sample sizes and nonlinear prediction problems; moreover, relative to CSA-LSSVM and single LSSVM, the proposed model shows higher fitting accuracy. The effect of fault identification of the model also presents significant superiority compared with RBF neural network techniques. This also indicates that it is feasible to identify the mechanical faults of OLTCs by applying CSA-fuzzy weighted LSSVM.

\section{Conclusion}

A CSA-fuzzy weighted LSSVM is constructed to identify mechanical faults in OLTCs. The model assigns different
TABLE 4: Confusion matrix of CSA-LSSVM.

\begin{tabular}{cccccc}
\hline & \multicolumn{5}{c}{ Predicted working } \\
& & & \\
& & 1 & 2 & 3 & 4 \\
\hline \multirow{3}{*}{ Actual working condition } \\
& 1 & 50 & 0 & 0 & 0 \\
& 2 & 2 & 47 & 1 & 0 \\
& 3 & 1 & 1 & 48 & 0 \\
& 4 & 1 & 1 & 1 & 47 \\
\hline
\end{tabular}

TABle 5: Confusion matrix of CSA-fuzzy weighted LSSVM.

\begin{tabular}{cccccc}
\hline & \multicolumn{5}{c}{ Predicted working } \\
& 1 & 2 & 3 & 4 \\
\hline \multirow{3}{*}{ Actual working condition } \\
& 1 & 50 & 0 & 0 & 0 \\
& 2 & 0 & 49 & 1 & 0 \\
& 3 & 0 & 1 & 49 & 0 \\
& 4 & 0 & 0 & 0 & 50 \\
\hline
\end{tabular}

TABLE 6: Statistical results showing classification accuracy of the four pattern recognition methods.

\begin{tabular}{lccc}
\hline Model & $\begin{array}{c}\text { Correctly } \\
\text { classified sample } \\
\text { size }\end{array}$ & $\begin{array}{c}\text { Classification } \\
\text { accuracy (\%) }\end{array}$ & Kappa \\
\hline $\begin{array}{l}\text { RBF neural } \\
\text { network }\end{array}$ & $137 / 200$ & 68.5 & 0.580 \\
$\begin{array}{l}\text { Single LSSVM } \\
\text { CSA-LSSVM }\end{array}$ & $177 / 200$ & 88.5 & 0.836 \\
$\begin{array}{l}\text { CSA-fuzzy } \\
\text { weighted }\end{array}$ & $192 / 200$ & 96.0 & 0.947 \\
LSSVM & $198 / 200$ & 99.0 & 0.987 \\
\hline
\end{tabular}

weights to the training data in different periods. Additionally, the parameters of the model are optimised by applying CSA to improve the accuracy of fault identification. The following conclusions are drawn:

(1) The mechanical state of an OLTC during long-term operation gradually changed and the characteristics of vibration signals measured in different periods also differed, therefore proving the importance of the characteristic data of vibration signals of OLTCs changes in different periods during the training phase. Assigning different weights to the training data in different periods can improve the identification accuracy of mechanical faults of OLTCs.

(2) Wavelet packet energy entropy can effectively characterise the frequency composition information of vibration signals of OLTCs at different scales and can describe the dynamic characteristics contained in vibration signals on the basis of decomposing the mechanical vibration signals of OLTCs. Therefore, wavelet packet energy entropy is suitable for extracting the features of mechanical vibration signals arising from OLTCs.

(3) The CSA-fuzzy weighted LASSVM can process small sample sizes and nonlinear classification 
TABLE 7: Abbreviations.

\begin{tabular}{ll}
\hline Term & Abbreviation \\
\hline Fast Fourier transform & FFT \\
Adaptive genetic algorithm & AGA \\
Least square support vector machine & LSSVM \\
Cuckoo search algorithm & CSA \\
Artificial neural network & ANN \\
Backpropagation & BP \\
Genetic algorithm & GA \\
On-load tap changer & OLTC \\
On-load tap changing transformer & OLTCT \\
Radial basis function & RBF \\
\hline
\end{tabular}

problems. Compared with CSA-LSSVM, single LSSVM, and RBF neural network methods, the model exhibits higher fitting accuracy and is applicable to diagnosis of mechanical faults in OLTCs.

The proposed diagnosis method would change the traditional diagnosis method of the on-load tap changer and improve the power supply quality and the detection efficiency under the premise of ensuring the safety of the staff.

However, the research in this article still has deficiencies. For example, this article does not conduct an in-depth study on the propagation law of the mechanical vibration waves of the on-load tap changer, nor does it take into account the noise of the core and winding vibrations during normal operation of the transformer. We need to do further research in the next step as follows:

(1) Based on the in-depth study of the internal structure of the on-load voltage-regulating transformer, the propagation law and attenuation mode of the mechanical vibration of the on-load tap changer in the transformer could be studied from the perspective of physical acoustics. The vibration sensor measurement method and vibration signal feature extraction method should be further improved.

(2) The vibration generation mechanism of transformer core and winding and other components should be studied, and we should pay attention to the application of blind source separation algorithm in extracting mechanical vibration to obtain more accurate and true mechanical vibration signal of onload tap changer.

The abbreviations are given in Table 7.

\section{Data Availability}

The data that support the findings of this study are available from State Grid Hebei Electric Power Research Institute but restrictions apply to the availability of these data, which were used under license for the current study, and so are not publicly available. Data are however available from the authors upon reasonable request and with permission of State Grid Hebei Electric Power Research Institute.

\section{Conflicts of Interest}

The authors declare that they have no conflicts of interest.

\section{Acknowledgments}

This work was supported by the State Grid Corporation of China Research Program "Research on Key Technologies of Condition-based Maintenance System Based on Noninterrupted Detection" (5204BB180002).

\section{References}

[1] P. Kang and D. Birtwhistle, "Characterisation of vibration sigsnals using continuous wavelet transform for condition assessment of on-load tap-changers," Mechanical Systems and Signal Processing, vol. 17, no. 3, pp. 561-577, 2003.

[2] P. Kang and D. Birtwhistle, "Condition assessment of power transformer on-load tap-changers using wavelet analysis and self-organizing map: field evaluation," Power Engineering Review, IEEE, vol. 22, no. 8, p. 69, 2007.

[3] E. Rivas, J. C. Burgos, and J. C. Garcia-Prada, "Condition assessment of power OLTC by vibration analysis using wavelet transform," IEEE Transactions on Power Delivery, vol. 24, no. 2, pp. 687-694, 2009.

[4] P. Kang and D. Birtwhistle, "Condition assessment of power transformer on-load tap-changers using wavelet analysis," IEEE Transactions On Power Delivery, vol. 16, no. 3, p. 394, 2001.

[5] Q. Li, T. Zhao, L. Zhang, and J. Lou, "Mechanical fault diagnostics of onload tap changer within power transformers based on hidden markov model," IEEE Transactions on Power Delivery, vol. 27, no. 2, pp. 596-601, 2012.

[6] X. L. Shi, "Study on mechanical fault diagnosis strategy of onload tap-changer based on characteristics of vibration signal," Henan Polytechnic University, Jiaozuo, China, Master Degree, 2017.

[7] F. M. Kong, Y. Chen, W. H. Li et al., "Research on mechanical fault diagnosis of high voltage circuit breakers based on particle swarm optimization PCA-LSSVM," Grid and Clean Energy, vol. 35, no. 10, pp. 68-74, 2019.

[8] M. Huang, D. Tian, H. Liu et al., "A hybrid fuzzy wavelet neural network model with self-adapted fuzzy C-means clustering and genetic algorithm for water quality prediction in rivers," Complexity, vol. 2018, Article ID 8241342, 11 pages, 2018.

[9] S. N. Syed Nasir, J. J. Jamian, and M. W. Mustafa, "Minimizing harmonic distortion impact at distribution system with considering large-scale EV load behaviour using modified lightning search algorithm and pareto-fuzzy approach," Complexity, vol. 2018, Article ID 6587493, 14 pages, 2018.

[10] J. Zhou and Q. Zhang, "Adaptive fuzzy control of uncertain robotic manipulator," Mathematical Problems in Engineering, vol. 2018, Article ID 4703492, 10 pages, 2018.

[11] G. Q. Ji, S. X. Li, W. B. Zhao et al., "Regional medium- and long-term power demand forecasting based on log-average dirichlet index-cuckoo search algorithm-least square support vector machine," Science Technology and Engineering, vol. 18, no. 10, pp. 213-218, 2018.

[12] X. Yang and S. Deb, "Cuckoo search via lévy flights," in Proceeding of the World Congress On Nature and Biologically Inspired Computing, IEEE, Coimbatore, India, pp. 210-214, December 2009. 
[13] X. Yand and S. Deb, "Engineering optimization by cuckoo search," International Journal of Mathematical Modeling and Numerical, vol. 1, no. 4, pp. 330-343, 2010.

[14] X. S. Yang, Nature-Inspired Metaheuristic Algorithms, Luniver Press, Beckington, UK, 2008.

[15] J. Ding, Q. Wang, Q. Zhang, Q. Ye, and Y. Ma, "A hybrid particle swarm optimization-cuckoo search algorithm and its engineering applications," Mathematical Problems in Engineering, vol. 2019, Article ID 5213759, 12 pages, 2019.

[16] L. Ding, X. Li, Q. Li, and Y. Chao, "Nonlinear friction and dynamical identification for a robot manipulator with improved cuckoo search algorithm," Journal of Robotics, vol. 2018, Article ID 8219123, 10 pages, 2018.

[17] J. GarcíaGarcía, A. F. Peña, O. Peña, G. Astorga, and O. Peredo, "A binary cuckoo search big data algorithm applied to large-scale crew scheduling problems," Complexity, vol. 2018, Article ID 8395193, 15 pages, 2018.

[18] T. T. Nguyen, C.-T. Nguyen, L. Van Dai, and N. Vu Quynh, "Finding optimal load dispatch solutions by using a proposed cuckoo search algorithm," Mathematical Problems in Engineering, vol. 2019, Article ID 1564693, 29 pages, 2019.

[19] N. Arunrat, C. Wang, and N. Pumijumnong, "Reprint of Alternative cropping systems for greenhouse gases mitigation in rice field: a case study in Phichit province of Thailand," Journal of Cleaner Production, vol. 134, pp. 547-562, 2016.

[20] M. Yang and Y. Yang, "Short-term photovoltaic output power prediction based on wavelet packet and LSSVM," Renewable Energy Resources, vol. 37, no. 11, pp. 1595-1602, 2019.

[21] L. Wang, Y. Q. Guo, and H. Sun, "Performance analysis and parameter optimization of aero-engine fault diagnosis," Aviation Computing Technology, vol. 47, no. 1, pp. 39-46, 2017. 